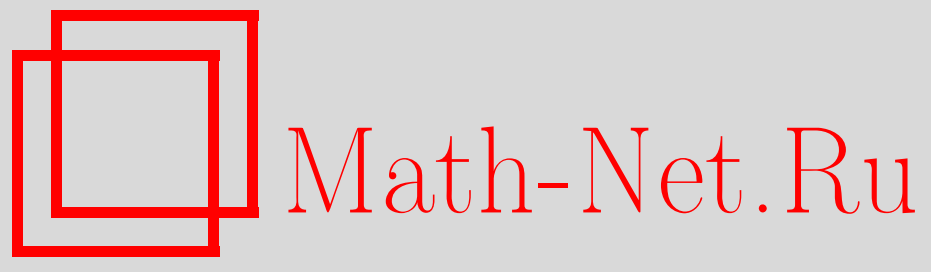

Ф. А. Шахиди, О крайних точках множества бистохастических операторов, Матем. заметки, 2008, том 84, выпуск 3, 475-480

DOI: https://doi.org/10.4213/mzm5545

Использование Общероссийского математического портала Math-Net.Ru подразумевает, что вы прочитали и согласны с пользовательским соглашением http://www .mathnet.ru/rus/agreement

Параметры загрузки:

IP : 54.209 .52 .79

26 апреля 2023 г., 10:26:48

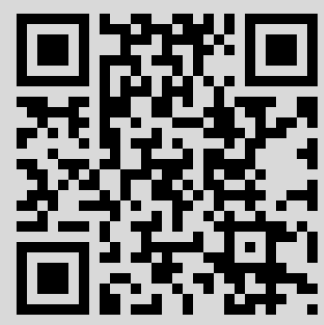




\section{О крайних точках множества бистохастических операторов}

\section{Фаррух Шахиди}

В работе [1] выделен класс квадратичных стохастических операторов (КСО), отображающих конечномерный симплекс в себя, которые называются бистохастическими квадратичными операторами (БКО). БКО тесно связаны с понятием мажоризации и применяются не только в задачах популяционной генетики [1], [2], но и в задачах экономики [3]. В математической экономике БКО называется оператором благосостояния. Изучение БКО было начато в работе [1] и было получено необходимое и достаточное условие для бистохастичности оператора. Эту теорему мы приведем ниже и неоднократно будем ею пользоваться. В данной работе показано, что множество БКО является выпуклым многогранником. Поэтому представляет интерес аналог теоремы Биркгофа о крайних точках множества бистохастических матриц [4]. В данной работе задача частично решена, а точнее, получено достаточное условие для крайности, а в двумерном симплексе получено необходимое и достаточное условие. Более того определено количество крайних точек множества БКО в двумерном симплексе. Теперь перейдем к необходимым понятиям.

ОПредЕЛЕние 1 [2]. Пусть $S^{m-1}-(m-1)$-мерный симплекс. Оператор $V: S^{m-1} \rightarrow$ $S^{m-1}$ называется квадратично стохастическим, если

$$
(V x)_{k}=\sum_{i, j=1}^{m} p_{i j, k} x_{i} x_{j}, \quad k=1, \ldots, m,
$$

где $x=\left(x_{1}, x_{2}, \ldots, x_{m}\right) \in S^{m-1}$ и

$$
p_{i j, k}=p_{j i, k} \geqslant 0 \quad \forall i, j, k=1, \ldots, m, \quad \sum_{k=1}^{m} p_{i j, k}=1 \quad \forall i, j=1, \ldots, m .
$$

Для каждого вектора $x=\left(x_{1}, x_{2} \ldots, x_{m}\right) \in \mathbb{R}^{m}$ положим $x_{\downarrow}=\left(x_{[1]}, x_{[2]}, \ldots, x_{[m]}\right)$, где $x_{[1]} \geqslant x_{[2]} \geqslant \cdots \geqslant x_{[m]}-$ компоненты вектора $x$, упорядоченные по невозрастанию.

Говорят, что $x$ мажорируется $y$ (или $y$ мажсорирует $x$ ), и пишут $x \prec y$, если

1) $\sum_{i=1}^{k} x_{[i]} \leqslant \sum_{i=1}^{k} y_{[i]}, k=1, \ldots, m-1$,

2) $\sum_{i=1}^{m} x_{[i]}=\sum_{i=1}^{m} y_{[i]}$.

ОПРЕДЕЛЕНИЕ 2 [3]. КСО называется бистохастическим, если

$$
V x \prec x \quad \forall x \in S^{m-1} .
$$

Обозначим через $\mathbf{B}$ множество бистохастических операторов. Очевидно, что $\mathbf{B} \neq \varnothing$ (например, единичный оператор является бистохастическим).

Пусть $(V x)_{k}=\sum_{i, j=1}^{m} p_{i j, k} x_{i} x_{j}-\mathrm{KCO}$. Положим $A_{k}=\left(p_{i j, k}\right)_{i, j=1, \ldots, m} \forall k=1, \ldots, m$. Тогда оператор $(*)$ имеет следующий вид:

$$
V x=\left[\left(A_{1} x, x\right),\left(A_{2} x, x\right), \ldots,\left(A_{m} x, x\right)\right]
$$

и

$$
\sum_{i=1}^{m} A_{i}=E
$$

где $E$ - матрица, все элементы которой равны 1 . Из (**) следует, что матрицы $A_{k}$ неотрицательные и симметрические.

(C) Фаррух ШАхиди, 2008 
Введем обозначение

$$
V=\left(A_{1}\left|A_{2}\right| \cdots \mid A_{m}\right) .
$$

Для $\forall \alpha \subset I=\{1,2, \ldots, m\}$ пусть $|\alpha|-$ мощность множества $\alpha$.

Пусть $U$ - множество матриц, определенное следующим образом:

$$
U=\left\{A=\left(a_{i j}\right)_{i, j=1, \ldots, m}: a_{i j}=a_{j i} \geqslant 0, \sum_{i, j \in \alpha} a_{i j} \leqslant|\alpha|, \sum_{i, j \in I} a_{i j}=m\right\} .
$$

Теорема 1 [1]. Необходимым и достаточным условием для бистохастичности оператора $V$ является условие $A_{k} \in U \forall k=1, \ldots, m u \sum_{i=1}^{m} A_{i}=E$.

Выпуклая оболочка конечного непустого множества точек в пространстве $\mathbb{R}^{k}$ называется выпуклым многогранником. Каждое ограниченное непустое пересечение конечного числа замкнутых полупространств есть выпуклый многогранник [5].

\section{ПреДЛОЖЕНИЕ 1. Множество В является выпуклым многогранником.}

ДокАзАтЕльство. Каждому квадратичному оператору соответствует кубическая матрица, которую можно вложить в пространство $\mathbb{R}^{m^{3}}$. Учитывая условия $(* *)$, ее можно вложить в $\mathbb{R}^{m^{2}(m-1) / 2}$. Далее, каждое из условий $(* * *)$ определяет замкнутое полупространство в пространстве $\mathbb{R}^{m^{2}(m-1) / 2}$. Из теоремы 1 следует, что В состоит из пересечения этих полупространств. Очевидно, что данное пересечение не пусто и является ограниченным множеством в $\mathbb{R}^{m^{2}(m-1) / 2}$, ибо оно лежит в положительном ортанте и в гиперплоскости $\sum_{i, j, k=1}^{m} p_{i j, k}=m^{2}$. Следуя Грюнбауму [5], мы заключаем, что $\mathbf{B}-$ замкнутый многогранник.

Так как $\mathbf{B}$ - выпуклый многогранник, $\mathbf{B}$ состоит из выпуклой комбинации его крайних точек. Поэтому представляет интерес описание его крайних точек.

Пусть $W$ - выпуклое множество. Точка $x \in W$ называется крайней, если из $2 x=$ $x_{1}+x_{2}, x_{1}, x_{2} \in W$ следует, что $x=x_{1}=x_{2}$. Множество крайних точек обозначим через $\operatorname{extr} W$.

Множество $U$ является компактным выпуклым множеством. Стало быть, существуют крайние точки этого множества. Предварительно опишем крайние точки множества $U$.

Квадратная матрица называется матрищей перестановки, если в каждой ее строке и в каждом столбце стоит ровно одна единица, а все остальные элементы являются нулями.

Лемма 1. Пусть $P \in U$ и $P$ матрица перестановок. Тогда $P \in \operatorname{extr} U$.

Доказательство. Докажем от противного. Пусть $P \notin \operatorname{extr} U$; тогда существуют $A, B \in U, A \neq B$ такие, что $2 P=A+B$. Пусть $A=\left(a_{i j}\right)_{i, j=1, \ldots, m}, B=\left(b_{i j}\right)_{i, j=1, \ldots, m}$; тогда $a_{i j}+b_{i j}=0$ либо 2 .

Если $a_{i j}+b_{i j}=0$, то $a_{i j}=b_{i j}=0$.

Если $a_{i j}+b_{i j}=2$, то рассмотрим два случая.

i) $i=j$; тогда $a_{i i}+b_{i i}=2$. Так как $a_{i i}, b_{i i} \leqslant 1$, то $a_{i i}=b_{i i}=1$.

ii) $i \neq j$. Рассмотрим множество $\alpha=\{i, j\}$. Если $a_{i j}>1$, то $\sum_{k, l \in \alpha} a_{i j}>2$, что противоречит $A \in U$. Поэтому $a_{i j} \leqslant 1$.

Аналогично получаем, что $b_{i j} \leqslant 1$. Так как $a_{i j}+b_{i j}=2$, то $a_{i j}=b_{i j}=1$. Таким образом, $a_{i j}=b_{i j} \forall i, j=1, \ldots, m$.

Следовательно, $A=B$, что противоречит нашему предположению. Лемма доказана.

Следует отметить, что обратное утверждение неверно.

УтвеРждение 1. Если $A \in \operatorname{extr} U$ и не является матрищей перестановки, то $\exists \alpha_{0} \subset I$ такой, что $\sum_{i, j \in \alpha_{0}} a_{i j}=\left|\alpha_{0}\right|$. 
ДоказАтельство. Пусть $A$ не является матрицей перестановки. Тогда существует элемент $a_{i_{0} j_{0}} \notin\{1,0\}$. Так как $\sum_{i, j \in I} a_{i j}=m$, то $\exists a_{i_{1} j_{1}} \notin\{1,0\}$. Пусть $\sum_{\alpha} a_{i j}<|\alpha|$ $\forall \alpha \subset I$. Тогда определим матрицы $B=\left(b_{i j}\right)_{1, \ldots, m} C=\left(c_{i j}\right)_{1, \ldots, m}$ следующим образом:

$$
\begin{aligned}
& B=\left\{b_{i_{0} j_{0}}=a_{i_{0} j_{0}}+\varepsilon, b_{i_{1} j_{1}}=a_{i_{1} j_{1}}-\varepsilon, b_{i j}=a_{i j} \text { при остальных значениях } i, j\right\}, \\
& C=\left\{c_{i_{0} j_{0}}=a_{i_{0} j_{0}}-\varepsilon, c_{i_{1} j_{1}}=a_{i_{1} j_{1}}+\varepsilon, c_{i j}=a_{i j} \text { при остальных значениях } i, j\right\}
\end{aligned}
$$

для некоторого достаточно малого числа $\varepsilon$. Тогда не трудно заметить, что при $\sum_{\alpha} a_{i j}<$ $|\alpha| \forall \alpha \subset I$ имеем $\sum_{\alpha} b_{i j}<|\alpha| \forall \alpha \subset I$ и $\sum_{\alpha} c_{i j}<|\alpha| \forall \alpha \subset I$. Следовательно, $B, C \in U$ и $B \neq C, B+C=2 A$, т.е. $A$ не крайняя.

Опишем крайние точки при $m=3$. Обозначим

$$
M=\left(\begin{array}{ccc}
0 & 1 / 2 & 1 / 2 \\
1 / 2 & 0 & 1 / 2 \\
1 / 2 & 1 / 2 & 0
\end{array}\right)
$$

Теорема 2. Пусть $A \in U, A \neq M$. Имеем

$$
A \in \operatorname{extr} U \Leftrightarrow a_{i i}=1 \vee 0, \quad a_{i j}=1 \vee \frac{1}{2} \vee 0 .
$$

ДоказАтельство. Если $A \in \operatorname{extr} U$, то согласно утверждению 1 имеют место три случая:

i) $a_{11}+2 a_{12}+a_{22}=2$;

ii) $a_{22}+2 a_{23}+a_{33}=2$;

iii) $a_{11}+2 a_{13}+a_{33}=2$.

Каждый из случаев рассматривается аналогичным образом. Следовательно, мы будем рассматривать только один случай.

Пусть

$$
a_{11}+2 a_{12}+a_{22}=2
$$

Докажем, что $a_{11}, a_{22} \in\{1,0\}$. Пусть $a_{11} \notin\{1,0\} \Rightarrow$ либо $a_{12} \notin\{1,0\}$, либо $a_{22} \notin\{1,0\}$.

1) Пусть $a_{12} \notin\{1,0\}$. Положим

$$
A^{\prime}=\left(\begin{array}{ccc}
a_{11}-2 \varepsilon & a_{12}+\varepsilon & a_{13} \\
a_{12}+\varepsilon & a_{22} & a_{23} \\
a_{13} & a_{23} & a_{33}
\end{array}\right), \quad A^{\prime \prime}=\left(\begin{array}{ccc}
a_{11}+2 \varepsilon & a_{12}-\varepsilon & a_{13} \\
a_{12}-\varepsilon & a_{22} & a_{23} \\
a_{13} & a_{23} & a_{33}
\end{array}\right) .
$$

Значение $\varepsilon>0$ можно выбрать столь малым, что все элементы матриц $A^{\prime}, A^{\prime \prime}$ будут неотрицательными. Тогда, если $a_{11}+2 a_{13}+a_{33}<2$, то $A^{\prime}, A^{\prime \prime} \in U, A^{\prime} \neq A^{\prime \prime}, 2 A=A^{\prime}+A^{\prime \prime}$, но $A \in \operatorname{extr} U$, поэтому

$$
a_{11}+2 a_{13}+a_{33}=2 .
$$

Так как $a_{11}+2 a_{13}+a_{33}=2$, то либо $a_{13} \notin\{1,0\}$, либо $a_{33} \notin\{1,0\}$.

1а) $a_{13} \notin\{1,0\}$. Положим

$$
A^{\prime}=\left\{a_{11}^{\prime}=a_{11}-2 \varepsilon, a_{13}^{\prime}=a_{13}+\varepsilon\right\}, \quad A^{\prime \prime}=\left\{a_{11}^{\prime \prime}=a_{11}+2 \varepsilon, a_{13}^{\prime \prime}=a_{13}-\varepsilon\right\} .
$$

Очевидно, $2 A=A^{\prime}+A^{\prime \prime}$ и $\left.A \notin \operatorname{extr} U \Rightarrow 1 \mathrm{a}\right)$ не имеет место.

1б) $a_{33} \notin\{1,0\}$. Тогда положим

$$
A^{\prime}=\left\{a_{11}^{\prime}=a_{11}-\varepsilon, a_{33}^{\prime}=a_{33}+\varepsilon\right\}, \quad A^{\prime \prime}=\left\{a_{11}^{\prime \prime}=a_{11}+\varepsilon, a_{33}^{\prime \prime}=a_{33}-\varepsilon\right\}
$$

$\Rightarrow 2 A=A^{\prime}+A^{\prime \prime}$. Если $a_{22}+2 a_{23}+a_{33}<2$, то $A \notin \operatorname{extr} U$. Поэтому

$$
a_{22}+2 a_{23}+a_{33}=2 .
$$

Но так как $A \in U$, то условия (1)-(3) не выполняются одновременно, поэтому случай 1$)$ не имеет место. 
2) Пусть $a_{22} \notin\{1,0\}$. Тогда

$$
2 A=A^{\prime}\left\{a_{11}^{\prime}=a_{11}-\varepsilon, a_{22}^{\prime}=a_{22}+\varepsilon\right\}+A^{\prime \prime}\left\{a_{11}^{\prime \prime}=a_{11}+\varepsilon, a_{22}^{\prime \prime}=a_{22}-\varepsilon\right\} .
$$

Если $a_{22}+2 a_{23}+a_{33}<2$, то $A \notin \operatorname{extr} U$. Поэтому $a_{22}+2 a_{23}+a_{33}=2$. Пусть $a_{22}+2 a_{23}+$ $a_{33}=22$. Тогда либо $a_{23} \notin\{1,0\}$, либо $a_{33} \notin\{1,0\}$.

2a) $a_{23} \notin\{1,0\}$. Тогда

$$
2 A=A^{\prime}\left\{a_{22}^{\prime}=a_{22}-2 \varepsilon, a_{23}^{\prime}=a_{23}+\varepsilon\right\}+A^{\prime \prime}\left\{a_{22}^{\prime \prime}=a_{22}+2 \varepsilon, a_{23}^{\prime \prime}=a_{23}-\varepsilon\right\} .
$$

То есть $A \notin \operatorname{extr} U$.

2б) $a_{33} \notin\{1,0\}$. Тогда

$$
2 A=A^{\prime}\left\{a_{22}^{\prime}=a_{22}-\varepsilon, a_{33}^{\prime}=a_{33}+\varepsilon\right\}+A^{\prime \prime}\left\{a_{22}^{\prime \prime}=a_{22}+\varepsilon, a_{33}^{\prime \prime}=a_{33}-\varepsilon\right\} .
$$

Если $a_{11}+2 a_{13}+a_{33}<2$, то $A^{\prime}, A^{\prime \prime} \in U$ и $A \notin \operatorname{extr} U$. Поэтому $a_{11}+2 a_{13}+a_{33}=2$. Но (1)-(3) не выполняются одновременно. Отсюда можно делать вывод, что если $A \in$ $\operatorname{extr} U$, то $a_{11} \in\{1,0\}$. Случай $a_{22} \notin\{1,0\}$ рассматривается аналогично. Поэтому $a_{22} \in$ $\{1,0\}$.

Итак, если $A \in \operatorname{extr} U$, то имеет место 4 случая
1) $A=\left(\begin{array}{ccc}1 & 0 & a_{13} \\ 0 & 1 & a_{23} \\ a_{13} & a_{23} & a_{33}\end{array}\right)$;
2) $A=\left(\begin{array}{ccc}0 & 1 & a_{13} \\ 1 & 0 & a_{23} \\ a_{13} & a_{23} & a_{33}\end{array}\right)$;
3) $A=\left(\begin{array}{ccc}1 & 1 / 2 & a_{13} \\ 1 / 2 & 0 & a_{23} \\ a_{13} & a_{23} & a_{33}\end{array}\right)$;
4) $A=\left(\begin{array}{ccc}0 & 1 / 2 & a_{13} \\ 1 / 2 & 1 & a_{23} \\ a_{13} & a_{23} & a_{33}\end{array}\right)$.

Рассмотрим только один случай. Остальные случаи рассматриваются аналогично. Пусть

$$
A=\left(\begin{array}{ccc}
1 & 0 & a_{13} \\
0 & 1 & a_{23} \\
a_{13} & a_{23} & a_{33}
\end{array}\right) .
$$

Предположим, что $a_{33} \notin\{1,0\}$. Если $2 a_{13}+a_{23}<1$, то

$$
2 A=A^{\prime}\left\{a_{33}^{\prime}=a_{33}-2 \varepsilon, a_{13}^{\prime}=a_{13}+\varepsilon\right\}+A^{\prime \prime}\left\{a_{33}^{\prime \prime}=a_{33}+2 \varepsilon, a_{13}^{\prime \prime}=a_{13}-\varepsilon\right\}
$$

и $A \notin \operatorname{extr} U$.

Если $2 a_{23}+a_{33}<1$, то

$$
2 A=A^{\prime}\left\{a_{33}^{\prime}=a_{33}-2 \varepsilon, a_{23}^{\prime}=a_{23}+\varepsilon\right\}+A^{\prime \prime}\left\{a_{33}^{\prime \prime}=a_{33}+2 \varepsilon, a_{23}^{\prime \prime}=a_{23}-\varepsilon\right\} .
$$

Следовательно, $a_{13}+a_{33}=1$ и $a_{23}+a_{33}=1$. Но эти условия не выполняются одновременно. Поэтому $a_{33} \in\{1,0\}$. Но так как $a_{33} \neq 1$ ( $A$ не перестановочная матрица), то $a_{33}=0$. Тогда $a_{13}+a_{23}=1 / 2$. Если $0<a_{13}<1 / 2$, то $0<a_{23}<1 / 2$ и

$$
2 A=A^{\prime}\left\{a_{13}^{\prime}=a_{13}-\varepsilon, a_{23}^{\prime}=a_{23}+\varepsilon\right\}+A^{\prime \prime}\left\{a_{13}^{\prime \prime}=a_{13}+\varepsilon, a_{23}^{\prime \prime}=a_{23}-\varepsilon\right\} .
$$

Поэтому $a_{13}, a_{23} \in\{1 / 2,0\}$. В этом случае находим две крайние точки множества $U$ :

$$
\left(\begin{array}{ccc}
1 & 0 & 1 / 2 \\
0 & 1 & 0 \\
1 / 2 & 0 & 0
\end{array}\right) \quad \text { и } \quad\left(\begin{array}{ccc}
1 & 0 & 0 \\
0 & 1 & 1 / 2 \\
0 & 1 / 2 & 0
\end{array}\right) .
$$


Рассмотрев все остальные случаи получим следующее

Следствие 1. Имеем $|\operatorname{extr} U|=25$.

В работе [1; гл. 3] было высказано предположение, что оператор $V=\left(A_{1}\left|A_{2}\right| \cdots \mid A_{m}\right)$ является крайним для В тогда и только тогда, когда $A_{i} \in \operatorname{extr} U \forall i=1, \ldots, m$.

Teоpema 3. Пусть $V=\left(A_{1}|\cdots| A_{m}\right) \in \operatorname{extr} \mathbf{B}$. Тогда $V_{\pi}=\left(A_{\pi(1)}|\cdots| A_{\pi(m)}\right) \in \operatorname{extr} B$ для любой перестановки $\pi$ множества индексов $\{1,2, \ldots, m\}$.

ДоказАтельство. Пусть $V_{\pi} \notin \operatorname{extr} \mathbf{B}$. Тогда $\exists V^{\prime}, V^{\prime \prime} \in \mathbf{B}, V^{\prime} \neq V^{\prime \prime}$, такие, что $2 V_{\pi}=$ $V^{\prime}+V^{\prime \prime}$. Пусть $V^{\prime}=\left(A_{1}^{\prime}|\cdots| A_{m}^{\prime}\right), V^{\prime \prime}=\left(A_{1}^{\prime \prime}|\cdots| A_{m}^{\prime \prime}\right)$. Тогда $\left(2 A_{\pi(1)}-A_{1}^{\prime}-A_{1}^{\prime \prime}|\cdots| 2 A_{\pi(m)}\right.$ $\left.A_{m}^{\prime}-A_{m}^{\prime \prime}\right)=\mathbf{0}$. Так как матрицы $2 A_{\pi(i)}-A_{i}^{\prime}-A_{i}^{\prime \prime}$ симметричны, то $2 A_{\pi(i)}=A_{i}^{\prime}+A_{i}^{\prime \prime}$ и, следовательно, $2 A_{i}=A_{\pi^{-1}(i)}^{\prime}+A_{\pi^{-1}(i)}^{\prime \prime}$. Так как

$$
\sum_{i=1}^{m} A_{i}^{\prime}=\sum_{i=1}^{m} A_{i}^{\prime \prime}=E
$$

To

$$
\sum_{i=1}^{m} A_{\pi^{-1}(i)}^{\prime}=\sum_{i=1}^{m} A_{\pi^{-1}(i)}^{\prime \prime}=E .
$$

Положим $W^{\prime}=\left(A_{\pi^{-1}(1)}^{\prime}|\cdots| A_{\pi^{-1}(m)}^{\prime}\right)$ и $W^{\prime \prime}=\left(A_{\pi^{-1}(1)}^{\prime \prime}|\cdots| A_{\pi^{-1}(m)}^{\prime \prime}\right)$. Тогда $W^{\prime}, W^{\prime \prime} \in \mathbf{B}$, $W^{\prime} \neq W^{\prime \prime}$ и $2 V=W^{\prime}+W^{\prime \prime}$. Получаем противоречие $V \notin \operatorname{extr} \mathbf{B}$.

Теорема 4. Пусть $V=\left(A_{1}|\cdots| A_{m}\right)$. Если для любой перестановки $\pi$ любых $m-1$ элементов из $\{1,2, \ldots, m\} \quad A_{\pi(k)} \in \operatorname{extr} U, m o V \in \operatorname{extr} \mathbf{B}$.

ДоказАтельство. По теореме 3 достаточно доказать, что если $A_{1}, A_{2}, \ldots, A_{m-1} \in$ $\operatorname{extr} U$, то $V \in \operatorname{extr} \mathbf{B}$. Последнее утверждение докажем от противного. Пусть $V \notin \operatorname{extr} \mathbf{B}$. Тогда $\exists V^{\prime}, V^{\prime \prime} \in \mathbf{B}, V^{\prime} \neq V^{\prime \prime}$, такие, что $2 V=V^{\prime}+V^{\prime \prime}$. Пусть $V^{\prime}=\left(A_{1}^{\prime}|\cdots| A_{m}^{\prime}\right), V^{\prime \prime}=$ $\left(A_{1}^{\prime \prime}|\cdots| A_{m}^{\prime \prime}\right)$. Тогда

$$
\left(2 A_{1}-A_{1}^{\prime}-A_{1}^{\prime \prime}|\cdots| 2 A_{m}-A_{m}^{\prime}-A_{m}^{\prime \prime}\right)=\mathbf{0} .
$$

Следовательно, $2 A_{i}-A_{i}^{\prime}-A_{i}^{\prime \prime}=\mathbf{0} \forall i=1, \ldots, m$. Так как $A_{i} \in \operatorname{extr} U \forall i \geqslant 2$, то $A_{i}^{\prime}=A_{i}^{\prime \prime}$ $\forall i \geqslant 2$. Из равенства $\sum_{i=1}^{m} A_{i}^{\prime}=\sum_{i=1}^{m} A_{i}^{\prime \prime}=E$ получаем, что $A_{1}^{\prime}=A_{1}^{\prime \prime}$. Таким образом, $V^{\prime} \neq V^{\prime \prime}$, что противоречит тому, что $V \notin \operatorname{extr} \mathbf{B}$.

Следствие 2. Если $A_{i} \in \operatorname{extr} U \forall i=1, \ldots, m, m o V \in \operatorname{extr}$ В. Однако обратное утверждение неверно.

ПримеР. Рассмотрим оператор

$$
\begin{aligned}
x_{1}^{\prime} & =x_{1} x_{2}+x_{1} x_{3}+x_{2} x_{3}, \\
x_{2}^{\prime} & =x_{2}^{2}+x_{3}^{2}+x_{1} x_{3}, \\
x_{3}^{\prime} & =x_{1}^{2}+x_{1} x_{2}+x_{2} x_{3} .
\end{aligned}
$$

Этому оператору соответствуют матрицы

$$
\left(\begin{array}{ccc}
0 & 1 / 2 & 1 / 2 \\
1 / 2 & 0 & 1 / 2 \\
1 / 2 & 1 / 2 & 0
\end{array}\right), \quad\left(\begin{array}{ccc}
0 & 0 & 1 / 2 \\
0 & 1 & 0 \\
1 / 2 & 0 & 1
\end{array}\right), \quad\left(\begin{array}{ccc}
1 & 1 / 2 & 0 \\
1 / 2 & 0 & 1 / 2 \\
0 & 1 / 2 & 0
\end{array}\right) .
$$

Не трудно заметить, что две из них крайние (теорема 2). Тогда из теоремы 4 следует, что $V \in \operatorname{extr} \mathbf{B}$.

Являются ли условия теоремы 4 необходимыми и достаточными? В двумерном симплексе этот вопрос решается утвердительно. 
Теорема 5. Пусть $V=\left(A_{1}\left|A_{2}\right| A_{3}\right) \in \mathbf{B}$. Тогда и толъко тогда $V \in \operatorname{extr} \mathbf{B}$, когда по крайней мере две из трех матрии $A_{1}, A_{2}, A_{3}$ являются крайними в $\mathbf{U}$.

ДокАзАТЕльство. Для доказательства этой теоремы мы воспользуемся теоремой 3 из [6], которая гласит, что если $V \in \operatorname{extr} \mathbf{B}$, то все элементы матриц $A_{1}, A_{2}, A_{3}$ равны либо 0 , либо $1 / 2$, либо 1. Следовательно, если $V \in \operatorname{extr} \mathbf{B}$, то либо $A_{1}, A_{2}, A_{3} \in \operatorname{extr} \mathbf{U}$, либо $A_{1}, A_{2}, A_{3}=M$. В случаях, когда $A_{1}, A_{2}, A_{3} \in \operatorname{extr} \mathbf{U}$ и одна из матриц равна $M$, теорема доказана. Теперь остался лишь случай, когда некоторые из матриц $A_{1}, A_{2}, A_{3}$ равны $M$. Все они не могут равняться $M$, так как сумма этих матриц не дает нам $E$. Если две из этих трех матриц, допустим $A_{1}, A_{2}$, равны $M$, то легко можно доказать, что образующий оператор не является крайним для $\mathbf{B}$.

СледСтвиЕ 3. При $m=3|\operatorname{extr} \mathbf{B}|=222$.

Доказательство. Пусть $V \in \operatorname{extr} \mathbf{B}, V=\left(A_{1}\left|A_{2}\right| A_{3}\right)$. Из теоремы 4 следует, что $A_{2}, A_{3} \in \operatorname{extr} U$ с точностью до перестановки. Поэтому из $A_{1}+A_{2}+A_{3}=E$ следует, что либо $A_{1} \in \operatorname{extr} U$, либо $A_{1}=M$.

Пусть $A_{1} \in \operatorname{extr} U$. Надо выбрать те тройки крайних точек, сумма которых равна $E$. Количество таких троек равно 31 . В случае, когда $A_{1}=M$, количество крайних точек равно 6.

За счет перестановки заключаем, что $|\operatorname{extr} \mathbf{B}|=37 \times 3 !=222$.

Автор выражает искреннюю благодарность проф. Р. Н. Ганиходжаеву за поставленную задачу и постоянное внимание к работе.

\section{СПИСОК ЦИТИРОВАННОЙ ЛИТЕРАТУРЫ}

[1] Р. Н. Ганиходжаев, Исследования по теории квадратичных стохастических операторов, Дис. ... докт. физ.-матем. наук, Ташкент, 1993. [2] Ю. И. Любич, Математические структуры в популяционной генетике, Наукова Думка, Киев, 1983. [3] А. Маршалл, И. Олкин, Неравенства: Теория мажсоризации и ее приложения, Мир, М., 1983. [4] G. Birkhoff, "Tres observaciones sobre el algebra lineal", Univ. Nac. Tucumán. Revista A, 5 (1946), 147-151. [5] B. Grünbaum, Convex Polytopes, Interscience Publ. John Wiley \& Sons, Inc., New York, 1967. [6] Р. Н. Ганиходжаев, УМH, 48:4 (1993), 231-232.

\section{Фаррух Шахиди}

Национальный университет Узбекистана

E-mail: farruh.shahidi@gmail.com
Поступило

05.03.2007

Исправленный вариант

05.02 .2008 